\title{
Stratification of ST-elevation myocardial infarction patients based on soluble CD40L longitudinal changes
}

\author{
PATRÍCIA NAPOLEÃO, LUÍS B. P. CABRAL, MAFALDA SELAS, CLÁUDIA FREIXO, \\ MARIA DO CÉU MONTEIRO, MARIA BEGOÑA CRIADO, MARINA C. COSTA, \\ FRANCISCO J. ENGUITA, ANA MARIA VIEGAS-CRESPO, CARLOTA SALDANHA, \\ MIGUEL MOTA CARMO, RUI CRUZ FERREIRA, and TERESA PINHEIRO
}

LISBOA AND PENAFIEL, PORTUGAL

\begin{abstract}
Involvement of soluble CD40 ligand (SCD40L) in thrombosis and inflammation on the context of coronary artery disease is currently being revised. In that perspective, we had studied the association of SCD4OL with markers of platelet activation and markers of endothelial and vascular function. On that cohort, a stratification of patients with acute myocardial infarction (AMI) 1 month after percutaneous coronary intervention $(\mathrm{PCl})$ was observed based on concentrations of SCD40L. The study intended to identify the groups of AMI patients with different profiles of SCD40L concentrations and verify how medication, clinical evolution, biochemical data, and markers of regulation of endothelial function at genetic (endothelial nitric oxide synthase polymorphisms) and post-transcriptional levels (circulating microRNAs) affect sCD40L serum levels. Lower quartiles of $\mathrm{SCD} 40 \mathrm{~L}(<2.3 \mathrm{ng} / \mathrm{mL})$ were associated with higher concentrations of $\mathrm{N}$-terminal pro-brain natriuretic peptide (NT-proBNP), high frequency of G894T polymorphism, and altered expression of a set of microRNAs assumed to be involved in the regulation of endothelial and cardiac function and myocardium hypertrophy, relative to patients in SCD40L upper quartiles. A characteristic SCD40L variation pattern in STEMI patients was identified. Low levels of SCD40L 1 month after PCl distinguish STEMI patients with worse prognosis, a compromised cardiac healing, and a persistent endothelial dysfunction, as given by the association between SCD40L, NT-proBNP, G894T polymorphism, and specific profile of miRNA expression. These results suggest SCD40L could have a prognostic value in STEMI patients. (Translational Research 2016;176:95-104)
\end{abstract}

\begin{abstract}
Abbreviations: $\mathrm{AMI}=$ acute myocardial infraction; $\mathrm{CAD}=$ coronary artery disease; eNOS = endothelial nitric oxide synthase; $\mathrm{miR}=$ microRNA; NT-proBNP $=\mathrm{N}$-terminal pro-brain natriuretic peptide; $\mathrm{PCl}=$ percutaneous coronary intervention; $\mathrm{SCD} 40 \mathrm{~L}=$ soluble CD40 ligand; $\mathrm{STEMI}=\mathrm{ST}$ segment elevation myocardial infraction
\end{abstract}

From the Carlota Saldanha Lab, Instituto Medicina Molecular (IMM), Faculdade de Medicina da Universidade de Lisboa, Lisboa, Portugal; IINFACTS-CESPU, Instituto de Investigação e Formação Avançada em Ciências e Tecnologias da Saúde, Penafiel, Portugal; Serviço Cardiologia, Hospital Santa Marta, Centro Hospitalar Lisboa Central (CHLC), Lisboa, Portugal; Maria Carmo-Fonseca Lab, Instituto Medicina Molecular (IMM), Faculdade de Medicina da Universidade de Lisboa, Lisboa, Portugal; Centro de Estudos do Ambiente e do Mar (CESAM) \& Departamento de Biologia Animal (DBA), Faculdade de Ciências da Universidade de Lisboa, Lisboa, Portugal; Centro de Estudos de Doenças Crónicas (CEDOC), NOVA Medical School, Lisboa, Portugal; Instituto de Bioengenharia e Biociências (IBB), Departamento de Engenharia e Ciências
Nucleares, Instituto Superior Técnico, Universidade de Lisboa, Lisboa, Portugal.

Submitted for publication December 18, 2015; revision submitted March 22, 2016; accepted for publication April 12, 2016.

Reprint requests: Patrícia Napoleão, Carlota Saldanha Lab, Instituto de Medicina Molecular, Av. Professor Egas Moniz, 1649-028 Lisboa, Portugal; e-mail: pnapoleao@medicina.ulisboa.pt.

1931-5244/\$ - see front matter

(C) 2016 Elsevier Inc. All rights reserved.

http://dx.doi.org/10.1016/j.trs1.2016.04.005 


\section{AT A GLANCE COMMENTARY}

Napoleão P, et al.

Background

- In a previous study evaluating the influence of sCD40L in endothelial and vascular function we found that time changes of $\mathrm{SCD} 40 \mathrm{~L}$ over 1 month after percutaneous coronary intervention (PCI) stratify STEMI patients;

- Influence of medication, clinical evolution, biochemical data, and markers of regulation of endothelial function at genetic and posttranscriptional levels on $\mathrm{SCD} 40 \mathrm{~L}$ was assessed.

\section{Translational Significance}

- For the first time, we found some patients display low $\mathrm{sCD} 40 \mathrm{~L}$ concentrations over time (low-sCD40L group) and others show an increase of sCD40L levels 1 month after PCI (high-sCD40L group);

- Patients in the low-sCD40L group were associated to high $\mathrm{N}$-terminal pro-brain natriuretic peptide and altered expression of 3 microRNAs involved in regulation of endothelial and cardiac function;

- Low-sCD40L group shows higher frequency of $\mathrm{T}$ allele of G894T polymorphism than in the high-sCD40L group;

- Low levels of sCD40L 1 month after PCI distinguish STEMI patients with worse prognosis, a compromised cardiac healing, and a persistent endothelial dysfunction;

- For STEMI patients, a prognostic value for sCD40L could be suggested.

\section{INTRODUCTION}

Current opinion suggests a differential role of soluble CD40 ligand (sCD40L) at different stages of coronary artery disease (CAD), contrasting with traditional view of an unvarying function of CD40L/CD40 system interactions in the disease. ${ }^{1}$ Cross-sectional studies have shown circulating levels of sCD40L relate to CAD and acute manifestations of the disease, such as, acute myocardial infarction (AMI). ${ }^{2-6}$ However, an unequivocal indication of its utility in chronic or acute CAD manifestations has not been obtained so far.

In the context of CAD, several in vitro studies evidenced the relevance of SCD40L in endothelial dysfunction, ${ }^{7-10}$ activation of different types of vascular cells, ${ }^{11}$ leukocyte trafficking, and homing, ${ }^{7,10}$ among other distinctive processes of the atherosclerotic pathology.
In a previous study conducted by us, ${ }^{5}$ we have studied association of sCD40L with markers of endothelial and vascular function and of platelet activation, after AMI. On that cohort, evidences were founded of a stratification of patients with ST segment elevation myocardial infarction (STEMI) based on SCD40L concentrations 1 month after percutaneous coronary intervention (PCI). The present study was designed to identify the groups of STEMI patients with different profiles of SCD40L concentrations and verify how medication, clinical evolution, biochemical data, and markers of regulation of endothelial function at genetic (endothelial nitric oxide synthase [eNOS] polymorphisms) and post-transcriptional levels (circulating microRNAs, which are key regulators of cardiac function ${ }^{12}$ ) affect sCD40L serum levels.

\section{MATERIALS AND METHODS}

Study groups. A consecutive sample of 200 subjects (57 women and 143 men) were recruited from the Cardiology Department and from the outpatient clinic of cardiovascular risk in Santa Marta Hospital (Centro Hospitalar de Lisboa Central, Lisbon, Portugal). Among those, 89 STEMI patients (with documented ST-elevation changes, creatine kinase $>3$ times normal and cardiac troponin $>0.01 \mathrm{ng} / \mathrm{mL}$ ), undergoing PCI as reperfusion therapy, were enrolled during the first 6 hours of onset of chest pain; and 48 age-matched patients with stable angina (SA), presenting typical chest discomfort and/or positive stress tests, which were submitted to coronary angiography. Furthermore, 63 healthy volunteers, the control group (CTR), with negative stress test, absence of any history of coronary disease, life-threatening diseases, or any other disease or condition that impairs compliance, were also recruited. These volunteers were not submitted to coronary angiography.

SA patients and CTR subjects were used as predictors of altered endothelial regulation in AMI.

Exclusion criteria included age $>85$ years, significant comorbidities as peripheral artery disease or carotid artery disease, known antecedents of malignant, infectious and concurrent inflammatory diseases, chronic renal insufficiency, and previous myocardial infarction in the last 5 years.

Laboratory blood analysis for clinical chemistry, including $\mathrm{N}$-terminal pro-brain natriuretic peptide (NT-proBNP), C-reactive protein (CRP) and cardiac troponin $\mathrm{T}(\mathrm{cTnT})$, were measured in all patients and controls (Table I).

Study protocol and follow-up evaluation. STEMI and SA patients were monitored at 3 time points as follows: day 0 before PCI and administration of therapy, such as 
Table I. Baseline characteristics of patients and controls, expressed as median and interquartile (Q25-Q75), unless otherwise indicated

\begin{tabular}{|c|c|c|c|}
\hline & $\operatorname{CTR}(n=63)$ & $S A(n=48)$ & STEMI $(n=89)$ \\
\hline \multicolumn{4}{|l|}{ Demographics } \\
\hline $\operatorname{Sex}(f / m)$ & $23 / 40$ & $13 / 35$ & $21 / 68$ \\
\hline Age (y) & $55(47-65)$ & $63(57-73)$ & $63(54-72)$ \\
\hline \multicolumn{4}{|l|}{ Risk factors/comorbidities ${ }^{*}$} \\
\hline Body mass index $\left(\mathrm{kg} / \mathrm{m}^{2}\right)$ & $26(24-28)$ & $28(25-29)$ & $27(24-30)$ \\
\hline Waist perimeter $(\mathrm{cm})$ & $86(82-94)$ & $96(91-102)$ & $99(89-106)$ \\
\hline Smoking, $\mathrm{n}(\%)$ & $4(6)$ & $4(8)$ & $39(44)^{+\neq}$ \\
\hline Dyslipidemia, n (\%) & $29(46)$ & $35(73)^{\dagger}$ & $47(53)^{\ddagger}$ \\
\hline Hypertension, n (\%) & $18(29)$ & $37(77)^{\dagger}$ & $58(65)^{\dagger}$ \\
\hline Diabetes mellitus, n (\%) & $2(3)$ & $16(33)^{\dagger}$ & $35(39)^{\dagger}$ \\
\hline \multicolumn{4}{|l|}{ Previous event medication } \\
\hline No medication, $\mathrm{n}(\%)$ & $46(73)$ & $5(10)^{\dagger}$ & $27(30)^{\dagger \neq}$ \\
\hline Aspirin, n (\%) & $4(6)$ & $32(67)^{\dagger}$ & $41(46)^{\dagger \neq}$ \\
\hline ACE-inhibitor, n (\%) & $9(14)$ & $24(50)^{\dagger}$ & $30(34)^{\dagger}$ \\
\hline$\beta$-blockers, $\mathrm{n}(\%)$ & $3(5)$ & $22(46)^{\dagger}$ & $26(29)^{\dagger \ddagger}$ \\
\hline Statins, $\mathrm{n}(\%)$ & $10(16)$ & $37(77)^{\dagger}$ & $35(39)^{\dagger \neq}$ \\
\hline \multicolumn{4}{|l|}{ Diagnostic markers } \\
\hline $\mathrm{CRP}(\mathrm{mg} / \mathrm{dL})$ & $3.1(1.2-3.5)$ & $3.2(1.6-5.9)$ & $6.30(3.2-13)$ \\
\hline $\mathrm{cTnT}(\mathrm{ng} / \mathrm{mL})$ & $<0.01^{\S}$ & $<0.01^{\S}$ & $0.47(0.07-3.6)^{\dagger \neq}$ \\
\hline NT-proBNP (pg/mL) & $38(16-64)$ & $98(51-247)$ & $356(145-1577)^{\dagger \neq}$ \\
\hline \multicolumn{4}{|l|}{ eNOS polymorphisms } \\
\hline G894T, GG/T (\%) & $38 / 62$ & $37 / 63$ & $38 / 63$ \\
\hline T786C, Tा/C (\%) & $43 / 57$ & $50 / 50$ & $28 / 72$ \\
\hline
\end{tabular}

Abbreviations: ACE-inhibitors, angiotensin-converting enzyme inhibitor; BMI, body mass index; CRP, C-reactive protein; CTnT, C-reactive protein and cardiac troponin T; eNOS, endothelial nitric oxide synthase; NT-proBNP; N-terminal pro-brain natriuretic peptide; SA, stable angina.

*Smoking: inhaled use of cigarettes, cigars, or pipes in any quantity, in the year previous to admission; dyslipidemia: total serum cholesterol $\geq 190 \mathrm{mg} / \mathrm{dL}$ or serum triglycerides $\geq 180 \mathrm{mg} / \mathrm{dL}$ or use of lipid-lowering medication; hypertension: systolic blood pressure $\geq 140 \mathrm{~mm} \mathrm{Hg}$ or diastolic, blood pressure $\geq 90 \mathrm{~mm} \mathrm{Hg}$ or use of antihypertensive therapy; diabetes mellitus: fasting plasma glucose concentration $\geq 7.0 \mathrm{mmol} / \mathrm{L}$ or $2 \mathrm{~h}$ plasma, glucose $\geq 11.1 \mathrm{mmol} / \mathrm{L}$ or confirmed as clinically known and treated diabetes, mellitus.

${ }^{\dagger} P<0.05$ versus CTR group.

$\ddagger P<0.05$ versus $S A$ group.

$\S$ Values below detection limit.

antithrombotic agents and IIb/IIIa inhibitors, and 2 and 30 days after PCI. This protocol was designed to evaluate AMI patients at acute phase of AMI (rupture and coronary occlusion) and at the early recovery phase (cardiac healing and left ventricular remodeling), 2 and 30 days later. All patients were medicated by an assistance clinician. Previous studies using this cohort indicated that influence of medication in the values of inflammatory markers lasted for several days after PCI. ${ }^{5,13}$ Therefore, patients' assessment 1 month after PCI represents the period of time for medication and clinical stabilization.

A follow-up was performed by consulting medical records and/or through telephone contact, for a period up to 1 year. Evaluated primary end point was cardiovascular death.

The study was conducted according to principles expressed in the Declaration of Helsinki and was approved by the Ethical Committee Board of Centro Hospitalar de Lisboa Central. All patients and volunteers enrolled signed a written consent following a full explanation of the study.

Blood sampling. Blood samples were drawn into blood collection tubes without additives. For STEMI and SA patients at the hospital, admission blood was collected immediately before PCI, which in case of STEMI patients was performed at hospital admission (within 6 hours of onset of chest pain). For subsequent time points and healthy volunteers (CTR), fasting blood samples were collected early in the morning to avoid possible circadian variations of measured parameters. ${ }^{14}$

Serum was collected after centrifugation $(500 \mathrm{~g}$ for 10 minutes). Aliquots were stored at $-80^{\circ} \mathrm{C}$ until further analysis (no longer than 6 months). Samples were thawed only once.

SCD40L measurement. Concentrations of SCD40L were measured in serum by enzyme-linked immunosorbent assay commercial kit (R\&D Systems). Each sample was measured in duplicate, and intra- 
assay variation among duplicates for all samples was $<10 \%$. Concentrations were expressed in nanogram per milliliter.

Having into consideration our previous exploratory analysis, ${ }^{5}$ serum was chosen to determine sCD40L by enzyme-linked immunosorbent assay. In addition, to safeguard sCD40L stability, temperature was kept at $4{ }^{\circ} \mathrm{C}$ in all steps after blood collection, that is, transport and processing. A rigorous protocol was applied to mitigate source of error originating from pre-analytical and analytical methodologies. 5

DNA extraction and genotyping. Polymorphisms G894T and T786C of eNOS protein were analyzed. Genomic DNA was extracted from peripheral blood cells collected in EDTA tubes using a PureLink Genomic DNA Mini Kit (Invitrogen). A region containing each polymorphism ${ }^{15}$ was amplified by polymerase chain reaction (PCR) using $1 \mathrm{mg}$ of DNA and $1 \mathrm{mM}$ of specific primers. Amplicons were then digested with specific restriction enzymes, and the digested fragments were visualized in a $2 \%$ ethidium bromide agarose gel.

G894T polymorphism corresponds to a modification in the coding sequence $\left(\mathrm{Glu}^{298} \rightarrow\right.$ Asp) located at the 894th position in exon 7 of the eNOS gene, which results in incorporation of aspartate in place of glutamate. ${ }^{16}$ T786C polymorphism result in a replacement of thymine by cytosine at nucleotide-786 position, decreasing transcription of eNOS gene and reducing in half the promoter activity. ${ }^{15}$

MicroRNA quantification analysis. RNA was extracted from serum samples using miRCURY RNA Isolation Kit (Exiqon). Complementary DNA was synthesized from RNA using Universal cDNA synthesis kit (Exiqon).

A microarray screening of circulating microRNAs was performed by quantitative PCR using a panel of 179 microRNAs (miRCURY LNA Universal RT microRNA PCR assays; Exiqon). Based on those results, 19 microRNAs were preselected, namely, miR-1, miR15b, miR-19b, miR-20b, miR-21, miR-27b, miR-32, miR-99a, miR-125b, miR-126, miR-133a, miR-145, miR-146, miR-147a, miR-155, miR-208b, miR-222, miR-382, and miR-485.

Expression of preselected microRNAs (LNA PCR primer, Exiqon) was assessed. Amplification was performed by quantitative real-time PCR followed by determination of melting curve according to the conditions as follows: $95^{\circ} \mathrm{C}$ for 10 minutes followed by 45 cycles of $95^{\circ} \mathrm{C}$ for 10 seconds and $60^{\circ} \mathrm{C}$ for 60 seconds. Triplicates were performed. Raw $\mathrm{Ct}$ values were normalized by global normalization method in each sample, and expression levels of all microRNAs calculated by the Delta-Delta-Ct method as implemented in
DataAssist v2 software. Fold changes $>2$ were considered significant. Results are expressed in relative quantification, which shows fold changes of a specific microRNA in 2 populations. ${ }^{17}$ Comparisons were as follows: CTR versus low-sCD40L group; CTR versus high-sCD40L group; low-sCD40L versus highsCD40L group; CTR versus SA; low-sCD40L group versus SA; and high-sCD40L group versus SA. For STEMI and SA patients, values were compared at hospital admission and 30 days later. A relative quantification value different than 1 indicates different expressions of a specific microRNA in 2 populations. ${ }^{17}$

Statistical analysis. Data were summarized as median and interquartiles (IQ) $25 \%$ and 75\% (Q25-Q75) for continuous variables and as proportions for categorical variables. Noncontinuous variables were analyzed using a $2 \times 2$ table and Fisher exact test and using a general linear model analysis of variance with Bonferroni correction for continuous variables.

As measures of blood markers for each STEMI and SA patient were not independent over time, a linear mixed effects (LME) model was applied. This statistical model describes longitudinal variations of each patient allowing to estimate differences in average slopes between baseline (hospital admission, day 0) and other time points, giving a measure of the variation of each blood marker over time. To apply LME, a logarithm transformation was applied to SCD40L.

LME model was also used to compare longitudinal variation between groups of patients (SA, STEMI and sCD40L-based STEMI groups and SA), and assess correlations between $S C D 40 L$ concentrations over time and other blood markers, demographics, clinical parameters, and therapy.

Values of $P<0.05$ were considered statistically significant. Calculations were performed using SPSS (version 22.0) and R (version 2.11.1) software.

\section{RESULTS}

STEMI patients' stratification based on SCD40L levels measured 30 days after PCI. Soluble SCD40L concentrations were reduced at baseline in STEMI and SA patients when compared with CTR. One month after PCI, sCD40L levels increased in SA patients to values similar to CTR, whereas in STEMI patients, they remained significantly diminished (Fig 1, A). These changes of $\mathrm{sCD} 40 \mathrm{~L}$ concentrations over time were significant in both STEMI and SA as previously reported by us. ${ }^{5}$

Two groups of patients were identified based on the $50 \%$ percentile of $\mathrm{sCD} 40 \mathrm{~L}$ concentrations measured 1 month after PCI (cutoff concentration of $2.26 \mathrm{ng} / \mathrm{mL}$ ). The low-sCD40L group describes cases with sCD40L concentrations below the $50 \%$ percentile (median; IQ; 

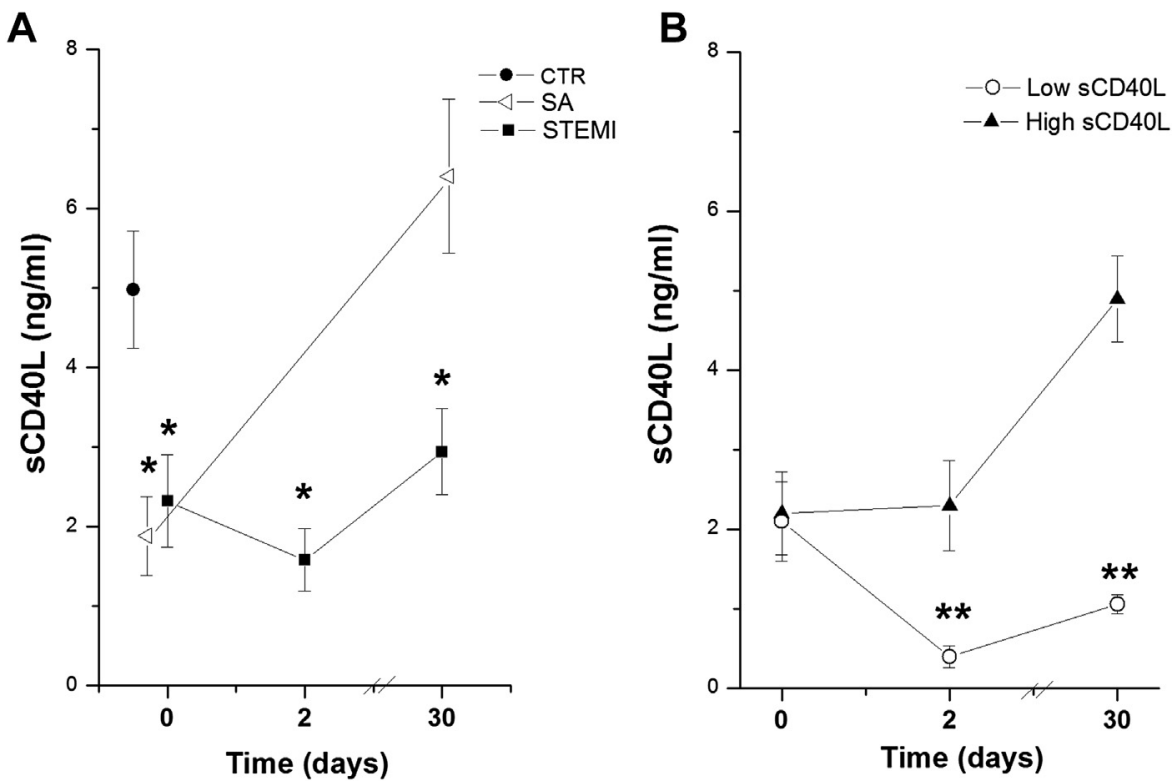

Fig 1. Variations of sCD40L levels over $1 \mathrm{mo}$ after PCI in studied populations (CTR group, SA and STEMI patients, A) and in STEMI groups, low- and high-sCD40L, based on SCD40L concentrations measured 1 mo after PCI (B). Significances $(P<0.05)$ versus CTR $(*)$ and versus low-sCD40L group $(* *)$ in each evaluation time point are indicated. PCI, percutaneous coronary intervention; SA, stable angina.

minimum-maximum: $0.88 ; 0.55-1.55 ; 0.36-2.26 \mathrm{ng} /$ $\mathrm{mL}$ ), whereas the high-sCD40L group describes cases with sCD40L levels above the $50 \%$ percentile (median; IQ; minimum-maximum: 3.48 ; 3.00-5.66; 2.33$11.2 \mathrm{ng} / \mathrm{mL}$ ). Soluble CD40L concentrations in highsCD40L group of STEMI patients were similar to SCD40L levels of CTR and of SA patients at day 30.

Modeling sCD40L average changes over time in these 2 groups of STEMI patients, a significant difference in longitudinal profile was observed $(\mathrm{F}=8.54$, $P<0.0001$ ) as can be inferred in Fig 1, $B$. Progressive average increase in sCD40L levels from hospital admission until day 30 in the high-sCD40L group contrasts with the average decrease in $\mathrm{SCD} 40 \mathrm{~L}$ after $\mathrm{AMI}$ in the low-sCD40L group.

High- and low-sCD40L groups did not differ in terms of number of patients, demographics, risk factors, and comorbidities (Table II). Influence of medication was addressed previously ${ }^{5}$ in this cohort, and no significant correlations between medication intake (pre-event and after hospital discharge) with sCD40L levels in serum were verified. As described in Table II, the 2 groups of STEMI patients revealed similar prescriptions; therefore, medicines do not contribute to stratification.

A 1-year clinical follow-up was considered having cardiovascular death as primary end point. In the overall of STEMI patients assessed, 5 patients died of cardiovascular causes (mean survival time of 4 months). Three of these patients died $<1$ month after PCI; conse- quently, they cannot account for SCD40L stratification. However, on those patients, sCD40L concentrations at day $2(0.77,0.68-1.02)$ were similar to those of the low-sCD40L group (Fig 1). Furthermore, the remaining 2 deaths (1 year after PCI) correspond to patients who were classified in the low-sCD40L group.

Longitudinal changes of NT-proBNP differed in SCD4OLbased STEMI groups. NT-proBNP showed significant changes in the 2 groups of STEMI patients (Table III). NT-proBNP concentrations 2 and 30 days after PCI significantly differ in both groups $(P<0.05)$. Concentrations of this natriuretic peptide remain high over 30 days in the low-sCD40L group contrasting with those in the high-sCD40L group, where levels sharply decrease to day 30 . Consequently, NT-proBNP time-variation trend over 30 days significantly differed in the low- and high-sCD40L groups $(\mathrm{F}=6.54$, $P=0.015)$. In addition, in STEMI patients, sCD40L increases were correlated with decreases in NT-proBNP concentrations.

eNOS genotypes in SCD40L-based STEMI groups. Frequency of polymorphisms of eNOS differed in the 2 sCD40L groups of STEMI patients (Fig 2). Regarding G894T polymorphism, T allele was more frequent in the low-sCD40L group (82\%) in comparison with that in the high-sCD40L group (33\%, $P<0.05)$. Frequency of T allele in the CTR group was diverse (Table I). No remarkable differences in frequency of eNOS T786C polymorphism were 
Table II. Characteristics of low- and high-sCD40L groups of STEMI patients

\begin{tabular}{|c|c|c|}
\hline & $\begin{array}{l}\text { Low-sCD40L } \\
(n=26)\end{array}$ & $\begin{array}{l}\text { High-sCD40L } \\
(n=25)\end{array}$ \\
\hline $\operatorname{Sex}(f / m)$ & $5 / 15$ & $2 / 18$ \\
\hline Age $(y)$ & $62(56-75)$ & $56(48-68)$ \\
\hline $\mathrm{BMI}\left(\mathrm{kg} / \mathrm{m}^{2}\right)$ & $27(24-31)$ & $28(25-31)$ \\
\hline Waist perimeter (cm) & $98(82-112)$ & $100(94-105)$ \\
\hline \multicolumn{3}{|l|}{ Risk factors/comorbidities } \\
\hline Smoking & $10(38)$ & $12(48)$ \\
\hline Dyslipidemia & $16(62)$ & $17(68)$ \\
\hline Hypertension & $17(65)$ & $20(80)$ \\
\hline Diabetes mellitus & $7(27)$ & $14(52)$ \\
\hline \multicolumn{3}{|l|}{ Medication-pre-event } \\
\hline No medication, n (\%) & $19(73)$ & $19(76)$ \\
\hline Aspirin, n (\%) & $19(35)$ & $13(52)$ \\
\hline ACE-inhibitor, n (\%) & $15(58)$ & $8(32)$ \\
\hline Anti-platelet inhibitors, n (\%) & $6(23)$ & $9(36)$ \\
\hline$\beta$-blockers, $\mathrm{n}(\%)$ & $6(23)$ & $11(44)$ \\
\hline Statins, n (\%) & $9(35)$ & $14(56)$ \\
\hline \multicolumn{3}{|l|}{ Medication_-discharge } \\
\hline Aspirin, n (\%) & $22(85)$ & $25(100)$ \\
\hline ACE-inhibitor, n (\%) & $18(69)$ & $22(88)$ \\
\hline Anti-platelet inhibitors, n (\%) & $124(92)$ & $25(100)$ \\
\hline$\beta$-blockers, n (\%) & $17(65)$ & $20(80)$ \\
\hline Statins, n (\%) & $22(85)$ & $25(100)$ \\
\hline
\end{tabular}

Abbreviations: ACE-inhibitors, angiotensin-converting enzyme inhibitor; $B M l$, body mass index.

Values are medians and interquartiles (Q25-Q75), unless indicated.

observed in both sCD40L-based STEMI groups and CTR.

MicroRNAs profiles in the SCD40L-based STEMI groups. MicroRNA quantification of 19 preselected microRNAs was performed in all samples of CTR, SA, and STEMI patients at days 0 and 30. Differences were found for miR-19b, miR-145, and miR-222 (Fig 3).

At hospital admission, STEMI patients did not show atypical microRNAs expression profile compared with that of the CTR group. However, 1 month after PCI, patients in sCD40L groups exhibited distinct microRNAs expression. No differences were verified in none of the 19 microRNAs quantified in the high-sCD40L group at day 30 versus CTR or SA. However, in the low-sCD40L group, miR-19b expression was 3-fold higher compared with that in CTR (Fig 3, A). Furthermore, at day 30, expression of miR-19b and miR-222 was both 4-fold higher in the low-sCD40L group comparing with SA patients (Fig 3, $A$ and $C$ ).

When low- and high-sCD40L groups were compared, striking differences in microRNA profile involving expression of miR-19b, miR-145, and miR-222 were observed (Fig 3). At day 30, expression of miR-19b, miR-145, and miR-222 in the low-sCD40L group was
Table III. Concentrations of CRP, CTnT, and NTproBNP measured at 3 time points (hospital admission-days 0,2 , and 30 after $\mathrm{PCl}$ ) in low- and high-sCD40L groups of STEMl patients

\begin{tabular}{lccc}
\hline & CRP $(\mathbf{m g} / \mathbf{d L})$ & cTnT $(\mathbf{n g} / \mathbf{m L})$ & NT-proBNP $(\mathbf{p g} / \mathbf{m L})$ \\
\hline Low-sCD4OL & & & \\
Day 0 & $4.3(3.2-7.4)$ & $0.85(0.07-2.9)$ & $356(158-1506)$ \\
Day 2 & $36(15-47)$ & $2.7(1.9-4.0)$ & $1567(851-3125)$ \\
Day 30 & $3.6(3.2-7.2)$ & $<0.01^{*}$ & $1090(625-2257)$ \\
High-sCD40L & & & \\
Day 0 & $5.2(3.2-9.3)$ & $0.24(0.06-3.5)$ & $175(80-1063)$ \\
Day 2 & $21(11-33)$ & $2.2(1.7-3.1)$ & $628(369-1423)^{\dagger}$ \\
Day 30 & $3.2(1.7-5.3)$ & $<0.01^{*}$ & $396(301-707)^{\dagger}$ \\
\hline
\end{tabular}

Abbreviations: CRP, C-reactive protein; CTnT, C-reactive protein and cardiac troponin T; NT-proBNP, N-terminal pro-brain natriuretic peptide; $\mathrm{PCl}$, percutaneous coronary intervention.

Values are median and interquartiles (Q25-Q75).

*Below detection limit.

${ }^{\dagger}$ Significantly different from the low-sCD4OL group, $P<0.05$.

4.5-6-fold increased comparing with the highsCD40L group (Fig 3).

Therefore, the low-sCD40L group was consistently associated with an altered post-transcriptional profile of microRNAs, whereas the high-sCD40L group showed similarities with CTR and SA.

\section{DISCUSSION}

This work showed sCD40L changes after PCI stratify STEMI patients, and these changes were associated with genetic and post-transcriptional markers. Results shown sCD40L concentrations 1 month after PCI stratify patients in 2 groups as follows: (1) a group of patients showing persistently low sCD40L concentrations over time (low-sCD40L group) and (2) a group of patients where sCD40L levels progressively increase to day 30 (high-sCD40L group).

Drug intake and clinical characteristics add no explanation to the stratification of STEMI patients. Nevertheless, in the low-sCD40L group, low sCD40L levels were linked to high concentrations of NT-proBNP, higher frequency of eNOS polymorphism G894T, and altered expression of microRNAs. This profile clearly departs from the high-sCD40L group, which shares similarities with CTR for sCD40L concentrations and microRNAs profile. The high-sCD40L group also showed comparable sCD40L longitudinal profile with SA patients 1 month after PCI.

Stratification of CAD patients based on SCD40L levels was previously proposed. ${ }^{4,18,19}$ Increased sCD40L levels have been used to identify patients with unstable $\mathrm{CAD}$ or at risk for acute syndromes, independently of other predictive variables. ${ }^{6,18,20}$ Apart from important methodology issues previously 
eNOS T786C
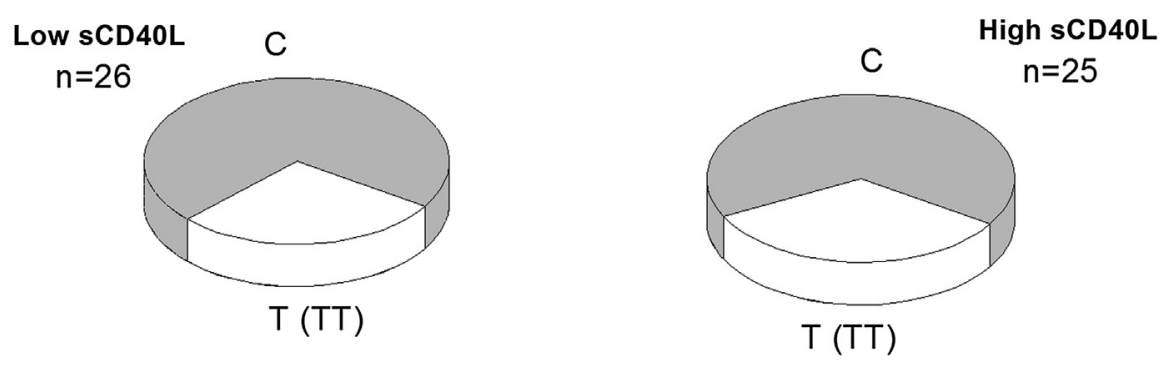

eNOS G894T
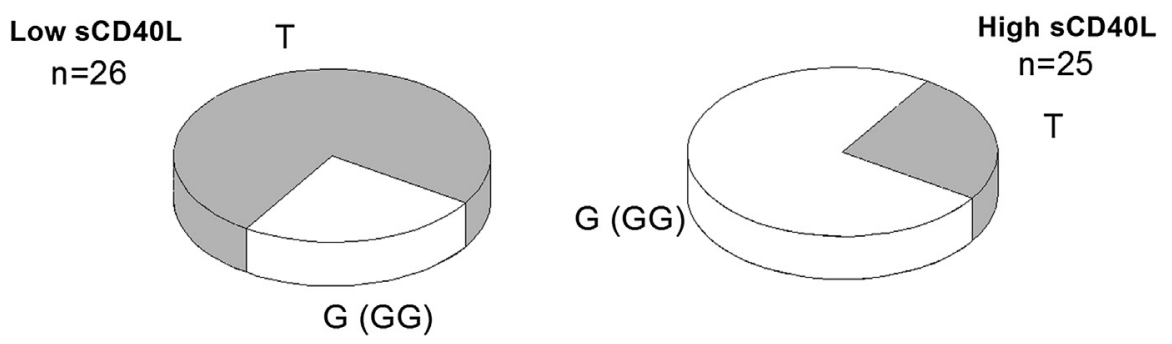

Fig 2. Variations of genotyping polymorphisms of eNOS T786C and G894T in low- and high-sCD40L groups of STEMI patients.
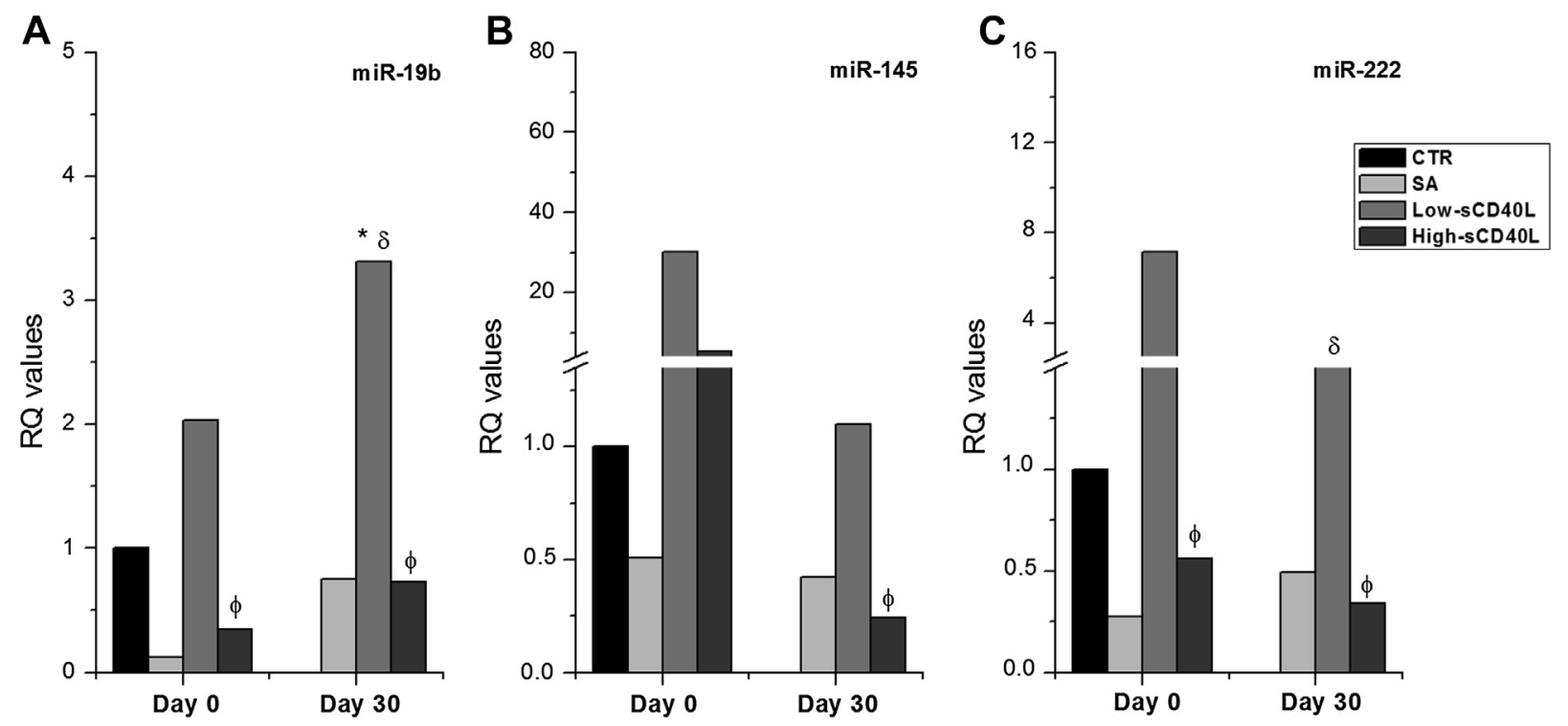

Fig 3. MicroRNAs miR-19b (A), miR-145 (B) and miR-222 (C) serum levels in CTR and SA groups and in sCD40L-based STEMI groups. Values represented are RQ values versus CTR group (reference group). ${ }^{*} P<0.05$ versus CTR, ${ }^{\delta} P<0.05$ versus SA group at day $30,{ }^{\varphi} P<0.05$ versus low-SCD40L group at day 30. $\mathrm{RQ}$, relative quantification; SA, stable angina.

mentioned by us, ${ }^{5}$ none of these studies exclusively assessed STEMI patients or variations of SCD40L over time as in the current work. Furthermore, more recent studies not only fail to demonstrate an association of high level of SCD40L with increased risk of cardiovas- cular events, ${ }^{21-24}$ but in fact, indicate an inverse relation of SCD40L levels with risk of recurrent ischemia. ${ }^{23}$

The CD40/CD40L system represents a major costimulator system triggering different signaling pathways mainly attending as amplification of immune 
and inflammatory responses, ${ }^{25}$ including in endothelial cells. $^{26,27}$ Therefore, CD40L can be implicated in promoting inflammation, ${ }^{9}$ angiogenesis, ${ }^{28}$ and endothelial dysfunction. ${ }^{7,29}$ Furthermore, overexpression of CD40 and CD40L was demonstrated in hearts of mice with myocarditis providing a mechanistic link between damaged myocardium and immune response. ${ }^{30}$

Soluble CD40L form also plays a role in CD40/ CD40L interaction between immune cells and nonimmune cells in a much wider context of the disease than thrombosis. ${ }^{5,31}$ Increases in solubilized CD40L may indicate lesser interaction with membrane-bound CD40 in target cells, either by release of CD40L exposed on cell surface or decreased expression of CD40L due to lack of extracellular and intracellular inhibitory stimulus of integrins and inflammatory molecules, ${ }^{31}$ rather than representing an increased costimulatory possibility. In fact, high affinity of sCD40L for CD40 when tumor necrosis factor alpha receptors and integrins are expressed was reported. ${ }^{32,33}$ This hypothesis finds an echo in the results obtained in our study, suggesting a detrimental effect of low sCD40L in AMI patients.

To our knowledge, this is the first clinical study directly linking sCD40L changes in the AMI evolution with post-transcriptional microRNAs profile. Previous in vitro and animal studies establish several microRNAs as having post-transcriptional regulator function of several pathways implicated in AMI. ${ }^{34,35}$ Among those are some microRNAs identified in the present study as upregulated in the low-sCD40L group versus controls such as miR-145. Previous studies positively related miR-145 to troponin $\mathrm{T}$ release establishing involvement of this microRNA in neointima repair in response to vascular injury. Thus, elevated miR-145 levels in the low-sCD40L group might be a result of vessel and myocardial injury as a consequence of MI. The miR-19b and miR-222 had also been related to endothelial and vascular dysfunction. ${ }^{36,37}$ MiR-222 has also been associated with vascular wound healing after injury and myocardium function and could contribute to heart diseases such as hypertrophic cardiomyopathy. ${ }^{37}$ Taking together, there seems to exist a profile of microRNAs related to endothelial dysfunction, compromised cardiac function, and myocardium hypertrophy associated with the low-sCD40L group, which is clearly different from controls, SA patients, and the high-sCD40L group.

Moreover, the low sCD40L group showed a higher frequency of T allele of G894T eNOS polymorphism. Prevalence of G894T eNOS polymorphism has been associated with physiological alterations and worse clinical outcomes, for example, low plasma NO concentrations and risk of CAD development. ${ }^{16,38}$ This polymorphism leads to a conservative replacement of glutamate with aspartate causing conformational alterations. ${ }^{39}$ Consequently, eNOS susceptibility to proteolytic cleavage in endothelial cells and vascular tissues is higher, which has a functional effect on the enzyme, ${ }^{39}$ and subsequently on NO bioactivity. ${ }^{39,40}$ Antoniades et $\mathrm{al}^{40}$ report that, in humans, G894T polymorphism may modify eNOS activity and is associated with endothelial dysfunction by decreasing NO but only under endothelial cell stimulation. According to those authors, the G894T polymorphism has no effect on the control group. ${ }^{40}$ These previous results suggest heterozygosity for the T allele could be associated with a compromised endothelial function in low-sCD40L STEMI patients, but not in the healthy subjects (CTR).

Finally, in our study, STEMI patients in the lowsCD40L group had higher NT-proBNP values, which are associated with adverse outcomes ${ }^{41-43}$ and is a clinically relevant marker of left ventricular dysfunction. ${ }^{44}$ Therefore, lower sCD40L concentrations can be associated with worse outcome and left ventricular dysfunction, as far as NT-proBNP variations are concerned.

In conclusion, our study shows STEMI patients with lower sCD40L levels have worse prognosis, a compromised myocardium function recovery, and a persistent endothelial dysfunction, as given by relationship between sCD40L, NT-proBNP, specific profile of microRNA expression, and frequency of G894T eNOS polymorphism. Results point out to involvement of sCD40L not only in thrombosis and inflammation but also in other important processes in cardiac, endothelial cells, and vascular system dysfunction, as we reported previously. ${ }^{5}$ This hypothesis could explain why the sCD40L-based stratification of STEMI patients was observed 1 month after PCI, in a period when the thrombotic effect is already diminished.

We hypothesize that in STEMI patients low sCD40L levels reflect a compromised recovery capacity of myocardium after infarct and persistent endothelial dysfunction. However, further mechanistic studies are mandatory to understand the molecular pathways involved in sCD40L-associated cardiac healing. Nevertheless, sCD40L could have a prognostic value in STEMI patients as a new biomarker of both endothelial and vascular function, being able to identify patients with higher risk of compromised cardiac healing.

\section{ACKNOWLEDGMENTS}

The work was financially supported by Fundação para a Ciência e Tecnologia (SFRM/BPD/6308/2009 and 


\section{UID/BIO/04565/2013) and Liga dos Amigos do Hospi- tal de Santa Marta. \\ Conflicts of Interest: All authors signing this article have read the journal's policy on conflicts of interest and have none to declare.}

\section{REFERENCES}

1. Zhang B, Wu T, Chen M, Zhou Y, Yi D, Guo R. The CD40/CD40L system: a new therapeutic target for disease. Immunol Lett 2013; 153:58-61.

2. Aukrust P, Müller F, Ueland T, et al. Enhanced levels of soluble and membrane-bound CD40 ligand in patients with unstable angina: possible reflection of T lymphocyte and platelet involvement in the pathogenesis of acute coronary syndromes. Circulation 1999;100:614-20.

3. Blankenberg S, McQueen MJ, Smieja M, et al. Comparative impact of multiple biomarkers and $\mathrm{N}$-terminal pro-brain natriuretic peptide in the context of conventional risk factors for the prediction of recurrent cardiovascular events in the heart outcomes prevention evaluation (HOPE) study. Circulation 2006; 114:201-8.

4. Kinlay S, Schwartz G, Olsson A, et al. Effect of atorvastatin on risk of recurrent cardiovascular events after an acute coronary syndrome associated with high soluble CD40 ligand in the myocardial ischemia reduction with aggressive cholesterol lowering (MIRACL) study. Circulation 2004;110:386-91.

5. Napoleão P, Monteiro MC, Cabral LB, et al. Changes of SCD40L in the progression of acute myocardial infarction associate to eNOS polymorphisms and VEGF but not to platelet CD26P expression. Transl Res 2015;166:650-9.

6. Varo N, de Lemos JA, Libby P, et al. Soluble CD40L: risk prediction after acute coronary syndromes. Circulation 2003;108: 1049-52.

7. Chen C, Chai H, Wang X, et al. Soluble CD40 ligand induces endothelial dysfunction in human and porcine coronary artery endothelial cells. Blood 2008;112:3205-16.

8. Furman MI, Krueger LA, Linden MD, Barnard MR, Frelinger AL III, Michelson AD. Release of soluble CD40L from platelets is regulated by glycoprotein $\mathrm{IIb} / \mathrm{III}$ a and actin polymerization. J Am Coll Cardiol 2004;43:2319-25.

9. Muhammad R, Pathak D, Freedman JE, Chakrabarti S. CD40CD40 ligand interactions in oxidative stress, inflammation and vascular disease. Trends Mol Med 2008;14:530-8.

10. Wolf D, Hohmann JD, Wiedemann A, et al. Binding of CD40L to Mac-1's I-domain involves the EQLKKSKTL motif and mediates leukocyte recruitment and atherosclerosis - but does not affect immunity and thrombosis in mice. Circ Res 2011;109:1269-79.

11. Hausding M, Jurk K, Daub S, et al. CD40L contributes to angiotensin II-induced pro-thrombotic state, vascular inflammation, oxidative stress and endothelial dysfunction. Basic Res Cardiol 2013; $108: 386$

12. Gurianova V, Stroy D, Ciccocioppo R, et al. Stress response factors as hub-regulators of microRNA biogenesis: implication to the disease heart. Cell Biochem Funct 2015;33:509-18.

13. Napoleão P, Selas M, Freixo C, et al. The role of inflammatory biomarkers in the assessment of coronary artery disease. In: Baskot B, ed. Coronary angiography - advances in noninvasive imaging approach for evaluation of coronary artery disease. Rijeka: InTech, 2011:281-314.

14. Dominguez-Rodriguez A, Abreu-Gonzalez P, GarciaGonzalez MJ, Kaski JC. Diurnal variation of soluble CD40 ligand in patients with acute coronary syndrome. Soluble CD40 ligand and diurnal variation. Thromb Res 2009;123:617-21.

15. Colombo MG, Paradossi U, Andreassi MG, et al. Endothelial nitric oxide synthase gene polymorphisms and risk of coronary artery disease. Clin Chem 2003;49:389-95.

16. da Costa Escobar Piccoli J, Manfredini V, Hamester FI, et al. Interaction between endothelial nitric oxide synthase gene polymorphisms (-786T $>\mathrm{C}, 894 \mathrm{G}>\mathrm{T}$ and intron $4 \mathrm{a} / \mathrm{b})$ and cardiovascular risk factors in acute coronary syndromes. Arch Med Res 2012;43:205-11.

17. Wang B, Wang XF, Xi Y. Normalizing bead-based microRNA expression data: a measurement error model-based approach. Bioinformatics 2011;27:1506-12.

18. Heeschen C, Dimmeler S, Hamm CW, et al. Soluble CD40 ligand in acute coronary syndromes. N Engl J Med 2003;348:1104-11.

19. Yan JC, Zhu J, Gao L, et al. The effect of elevated serum soluble CD40 ligand on the prognostic value in patients with acute coronary syndromes. Clin Chim Acta 2004;343:155-9.

20. Schönbeck U, Varo N, Libby P, Buring J, Ridker PM. Soluble CD40L and cardiovascular risk in women. Circulation 2001; 104:2266-8.

21. de Lemos JA, Zirlik A, Schönbeck U, et al. Associations between soluble CD40 ligand, atherosclerosis risk factors, and subclinical atherosclerosis: results from the Dallas Heart Study. Arterioscler Thromb Vasc Biol 2005;25:2192-6.

22. Lip GY, Patel JV, Hughes E, Hart RG. High-sensitivity C-reactive protein and soluble CD40 ligand as indices of inflammation and platelet activation in 880 patients with nonvalvular atrial fibrillation: relationship to stroke risk factors, stroke risk stratification schema, and prognosis. Stroke 2007;38: 1229-37.

23. Olenchock BA, Wiviott SD, Murphy SA, et al. Lack of association between soluble CD40L and risk in a large cohort of patients with acute coronary syndrome in OPUS TIMI-16. J Thromb Thrombolysis 2008;26:79-84.

24. Tanne D, Haim M, Goldbourt U, et al. CD40 ligand and risk of ischemic stroke or coronary events in patients with chronic coronary heart disease. Int J Cardiol 2006;107:322-6.

25. Elgueta R, Benson MJ, de Vries VC, Wasiuk A, Guo Y, Noelle RJ. Molecular mechanism and function of CD40/CD40L engagement in the immune system. Immunol Rev 2009;229: $152-72$.

26. Henn V, Steinbach S, Büchner K, Presek P, Kroczek RA. The inflammatory action of CD40 ligand (CD154) expressed on activated human platelets is temporally limited by coexpressed CD40. Blood 2001;98:1047-54.

27. Wagner AH, Güldenzoph B, Lienenlüke B, Hecker M. CD154/ CD40-mediated expression of CD154 in endothelial cells: consequences for endothelial cell-monocyte interaction. Arterioscler Thromb Vasc Biol 2004;24:715-20.

28. Melter M, Reinders MEJ, Sho M, et al. Ligation of CD40 induces the expression of vascular endothelial growth factor by endothelial cells and monocytes and promotes angiogenesis in vivo. Blood 2000;96:3801-8.

29. Hristov M, Gümbel D, Lutgens E, Zernecke A, Weber C. Soluble CD40 ligand impairs the function of peripheral blood angiogenic outgrowth cells and increases neointimal formation after arterial injury. Circulation 2010;121:315-24.

30. Seko Y, Takahashi N, Oshima H, et al. Expression of tumour necrosis factor (TNF) receptor/ligand superfamily co-stimulatory molecules CD40, CD30L, CD27L, and OX40L in murine hearts with chronic ongoing myocarditis caused by coxsackie virus B3. J Pathol 1999; 188:423-30. 
31. Otterdal K, Pedersen TM, Solum NO. Release of soluble CD40 ligand after platelet activation: studies on the solubilization phase. Thromb Res 2004;114:167-77.

32. André P, Prasad KS, Denis CV, et al. CD40L stabilizes arterial thrombi by a beta3 integrin-dependent mechanism. Nat Med 2002;8:247-52.

33. Zirlik A, Bavendiek U, Libby P, et al. TRAF-1, $-2,-3,-5$, and -6 are induced in atherosclerotic plaques and differentially mediate proinflammatory functions of CD40L in endothelial cells. Arterioscler Thromb Vasc Biol 2007;27:1101-7.

34. D'Alessandra Y, Devanna P, Limana F, et al. Circulating microRNAs are new and sensitive biomarkers of myocardial infarction. Eur Heart J 2010;31:2765-73.

35. Meder B, Keller A, Vogel B, et al. MicroRNA signatures in total peripheral blood as novel biomarkers for acute myocardial infarction. Basic Res Cardiol 2011;106:13-23.

36. Xue Y, Wei Z, Ding H, et al. MicroRNA-19b/221/222 induces endothelial cell dysfunction via suppression of PGC-1a in the progression of atherosclerosis. Atherosclerosis 2015;241:671-81.

37. Christiakov DA, Sobenin IA, Orekhov AN, Bobryshev YV. Human miR-221/222 in physiological and atherosclerotic vascular remodeling. Biomed Res Int 2015;354517:18.

38. Hingorani $\mathrm{AD}$, Liang $\mathrm{CF}$, Fatibene J, et al. A common variant of the endothelial nitric oxide synthase (Glu298/Asp) is a major risk factor for coronary artery disease in the UK. Circulation 1999; 100:1515-20.
39. Tesauro M, Thompson WC, Rogliani P, Qi L, Chaudhary PP, Moss J. Intracellular processing of endothelial nitric oxide synthase isoforms associated with differences in severity of cardiopulmonary diseases: cleavage of proteins with aspartate vs. glutamate at position 298. Proc Natl Acad Sci U S A 2000;97: 2832-5.

40. Antoniades C, Tousoulis D, Vasiliadou C, et al. Genetic polymorphism on endothelial nitric oxide synthase affects endothelial activation and inflammatory response during the acute phase of myocardial infarction. J Am Coll Cardiol 2005;46:1101-9.

41. Brueckmann M, Bertsch T, Lang S, et al. Time course of systemic markers of inflammation in patients presenting with acute coronary syndromes. Clin Chem Lab Med 2004;42:1132-9.

42. Navarro Estrada JL, Rubinstein F, Bahit MC, et al. NT-probrain natriuretic peptide predicts complexity and severity of the coronary lesions in patients with non-ST-elevation acute coronary syndromes. Am Heart J 2006;151:1093.e1-e7.

43. Sadanandan S, Cannon CP, Chekuri K, et al. Association of elevated B-type natriuretic peptide levels with angiographic findings among patients with unstable angina and non-ST-segment elevation myocardial infarction. J Am Coll Cardiol 2004;44: 564-8.

44. Valente S, Lazzeri C, Chiostri M, et al. NT-proBNP on admission for early risk stratification in STEMI patients submitted to PCI. Relation with extension of STEMI and inflammatory markers. Int J Cardiol 2009;132:84-9. 